\title{
The missing compact star of SN1987A: a solid quark star?
}

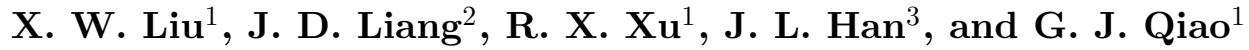 \\ ${ }^{1}$ School of Physics and State Key Laboratory of Nuclear Physics and Technology, \\ Peking University, Beijing 100871, China \\ email: xiongwliu@163.com \\ ${ }^{2}$ Yunnan Astronomical Observatory, Chinese Academy of Sciences, Kunming 650011, China \\ ${ }^{3}$ National Astronomical Observatories, Chinese Academy of Sciences, Beijing 100012, China
}

\begin{abstract}
To investigate the missing compact star of Supernova 1987A, we analyzed the cooling and heating processes of a possible compact star based on the upper limit of observational X-ray luminosity. From the cooling process, we found that a solid quark-cluster star (SQS), having a stiffer equation of state than that of a conventional liquid quark star, has a heat capacity much smaller than a neutron star. The SQS can cool down quickly, naturally explaining the nondetection of a point source in X-ray wavelengths. On the other hand, we considered the heating processes due to magnetospheric activity and possible accretion and obtained some constraints on the parameters of a possible pulsar. Therefore, we concluded that a SQS can explain the observational limit in a confident parameter space. As a possible central compact object, the pulsar parameter constraints can be tested for SN1987A with advanced, future facilities.
\end{abstract}

Keywords. pulsar: general - elementary particles - supernovae - star: neutron

\section{Introduction}

The missing compact star of Supernova 1987A is puzzling, mainly because of three factors: First, the neutrino burst from SN1987A indicates the forming of a neutron star; Second, the X-ray luminosity of a cooling neutron star is higher than the observational upper limit at $1 \mathrm{keV}$ (McCray 2007), thus the implied neutron star is expected to be detectable; and third, multi-wavelength observations did not find any point source or pulses at the position of SN1987A. Therefore, there must be something wrong or unusual.

There are two existing explanations for this puzzle: 1) The neutron star has transformed to a black hole via accretion from fall-back material; 2) It is a normal quark star, which cools very fast, that has luminosity lower than the observational limit (Chan et al. 2009). We note that both hypotheses have trouble explaining a 2 solar mass neutron star (Demorest et al. 2010), which means the maximum mass is larger than ever thought. The normal quark star is almost ruled out since its equation of state is too soft to reach 2 solar masses. And a black hole should be more difficult to form because it would need to accrete more fall-back material to exceed the maximum mass.

We propose that the compact star in SN1987A is a solid quark cluster star (SQS). The SQS has very small heat capacity thus cools faster than neutron star and has smaller X-ray luminosity than the observational limit, and it has stiff equation of state that can have maximum mass larger than 2 solar mass (Lai \& Xu 2009). 


\section{Cooling of quark cluster star}

\subsection{Heat capacity}

The heat capacity of quark cluster star is composed by the contributions of lattice structure $C_{\mathrm{SQS}}^{\mathrm{l}}$ and electrons $C_{\mathrm{SQS}}^{\mathrm{e}}$, i.e. $C_{\mathrm{SQS}}=C_{\mathrm{SQS}}^{\mathrm{l}}+C_{\mathrm{SQS}}^{\mathrm{e}}(\mathrm{Yu} \& \mathrm{Xu} 2011)$, where $C_{\mathrm{SQS}}^{\mathrm{l}}$ is proportional to $T^{3}$, and $C_{\mathrm{SQS}}^{\mathrm{e}}$ is proportional to $T$. The heat capacity is dominated by electrons when the temperature is lower than $\sim 10^{10} \mathrm{~K}$. The contribution of electrons is $C_{\mathrm{SQS}}^{\mathrm{e}} \simeq 3.5 \times 10^{37}\left(Y M_{1}\right)^{2 / 3} R_{6} T_{9}$ erg $\mathrm{K}^{-1}$ where $Y \sim 10^{-5}$ is the electron fraction.

\subsection{Cooling curve}

The heat energy could emit via neutrino radiation, color superconductivity related photon emission and bremsstrahlung. Figure 1 shows the cooling curve of a quark cluster star via only Bremsstrahlung (BR), compared with black-body emission. It shows that even if it cools only via bremsstrahlung the luminosity is lower than the observational limit at the age of 20 years.

\section{Restrict parameters via heating}

The heating luminosity should also be lower that the limit, thus it can be used to restrict the compact star's parameters.

\subsection{Heating from magnetosphere}

The rotational energy lost rate is related to the magnetic field and rotation frequency $\dot{E}=-\frac{2}{3 c^{3}} \mu_{\perp}^{2} \Omega^{4}$. The thermal X-ray luminosity is proportional to $\dot{E}$, i.e. $L_{b o l}^{\infty}=a \dot{E}$, where $a \sim 10^{-3}$ (Yu \& Xu 2011). The red lines in two panels of figure 2 are obtained with $a=10^{-4}, 10^{-3}$ and $10^{-2}$. The areas left to the lines should be ruled out.

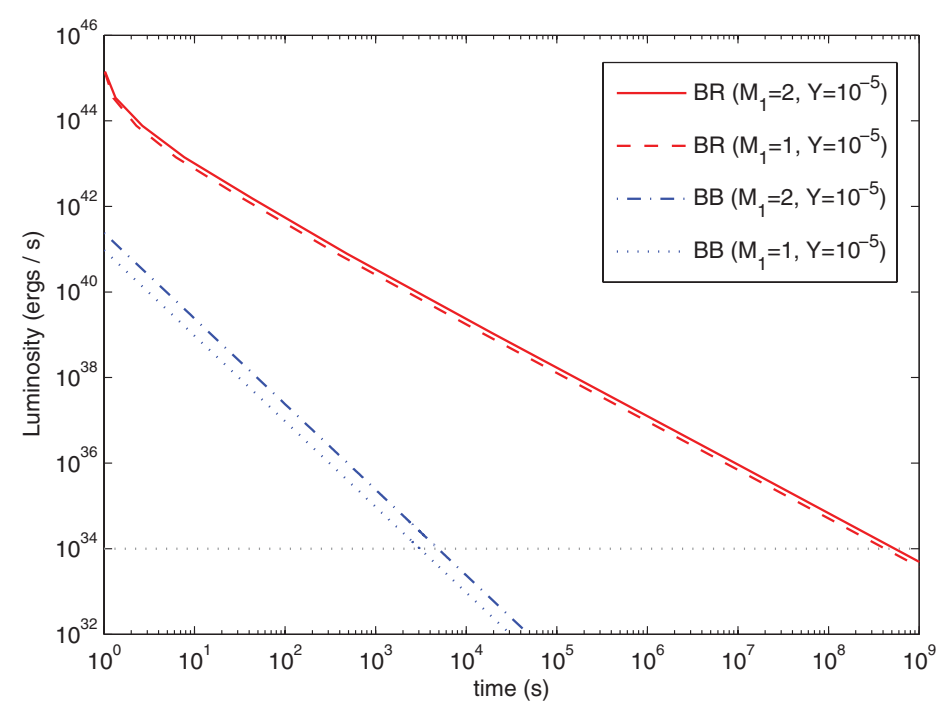

Figure 1. The cooling curves of SQSs with bremsstrahlung (BR) (Caron \& Zhitnitsky 2009) and black-body radiation (BB). Neutrino radiation and color superconductivity related photon emission are not considered. The observational upper limit is indicated as a horizontal dotted line. It shows that the cooling luminosity of a SQS could be smaller than $10^{34} \mathrm{ergs} \mathrm{s}^{-1}$ about 20 years after its birth even it cools down by bremsstrahlung emission. Here we take the stellar mass $M=M_{1} M_{\odot}$ and the number ratio of electron to baryon as $Y$. 

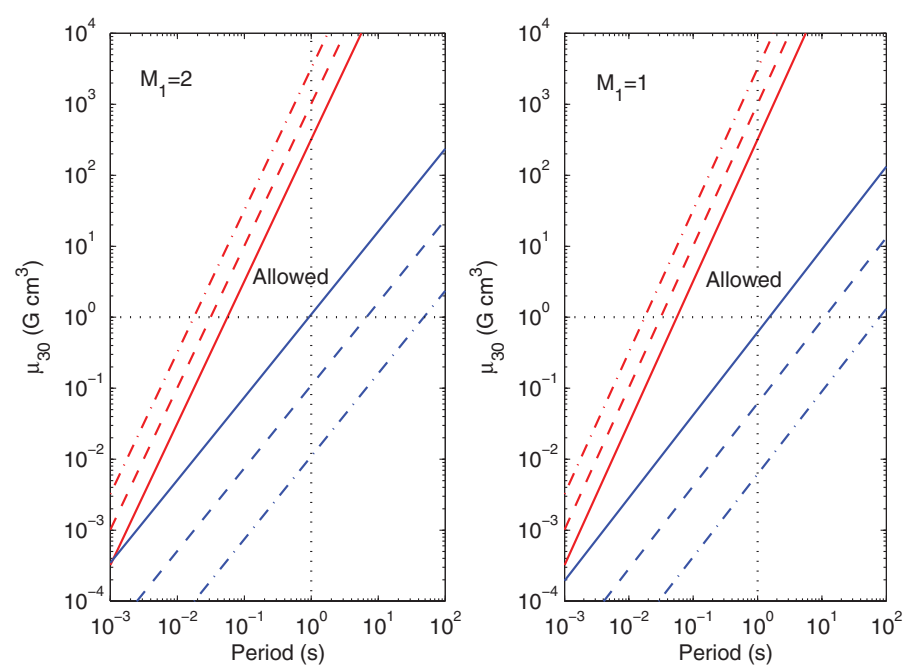

Figure 2. Constraints on parameters of the possible compact object in SN1987A via magnetosphere action heating (upper-left three lines) and fall back disk accretion heating (lower-right three lines). Left panel: $M_{1}=2$ and $b=0.9, a=10^{-4}$ for red dash-dotted line, $a=10^{-3}$ for red dashed line, $a=10^{-2}$ for red solid line, $\dot{M}=10^{18} \mathrm{erg} \mathrm{s}^{-1}$ for blue solid line, $\dot{M}=10^{16}$ erg $\mathrm{s}^{-1}$ for blue dashed line and $\dot{M}=10^{14} \mathrm{erg} \mathrm{s}^{-1}$ for blue dash-dotted line. Right panel: Same as the left but $M_{1}=1$.

\subsection{Heating from accretion}

If there is a disk around, it should not be in accretion phase which usually has very large luminosity. Thus $r_{m}>r_{b r}$, yielding $\mu_{30}>0.074 b^{7 / 4} M_{1}{ }^{5 / 6} \dot{M}_{16}^{1 / 2} P^{7 / 6}$. The blue lines in two panels of the figure 2 are obtained with $\dot{M}=10^{18} \mathrm{erg} \mathrm{s}^{-1}$ for blue solid line, $\dot{M}=10^{16} \mathrm{erg} \mathrm{s}^{-1}$ for blue dashed line and $\dot{M}=10^{14} \mathrm{erg} \mathrm{s}^{-1}$ for blue dash-dotted line.

\section{Summary}

Based on the foregoing analysis and calculation results, we conclude that:

1) A sold quark star with normal parameters is compatible with the non-detection in SN1987A. A low-mass quark cluster star has a wider parameter space than a more massive one;

2) If the surround dust is opacity in X-ray band, the parameter constraints become relaxed. The parameter space of a solid quark star is wider than that of a neutron star;

3) The parameter constraints are model independent.

As a possible central compact object, the parameters constrained for the pulsar can be tested for SN1987A with advanced, future facilities.

\section{References}

Caron, J.-F. \& Zhitnitsky, A. R. 2009, Phys. Rev. D, 80, 123006

Chan, T. C., Cheng, K. S., Harko, T., et al. 2009, ApJ, 695, 732

Demorest, P., Pennucci, T., Ransoml, S., Roberts, M., \& Hessels, J. W. T. 2010, Nature, 467, 1081

Lai, X. Y. \& Xu, R. X. 2009, MNRAS, 398, L31

McCray, R. 2007, AIP Conf. Proc., 937, 3

Yu, M. \& Xu, R. X. 2011, Astropart. Phys., 34, 493 